\title{
On the Partial Regularity of a 3D Model of the Navier-Stokes Equations
}

\author{
Thomas Y. Hou* Zhen Lei ${ }^{\dagger}$
}

August 13, 2008

\begin{abstract}
We study the partial regularity of a 3D model of the incompressible Navier-Stokes equations which was recently introduced by the authors in [11]. This model is derived for axisymmetric flows with swirl using a set of new variables. It preserves almost all the properties of the full 3D Euler or Navier-Stokes equations except for the convection term which is neglected in the model. If we add the convection term back to our model, we would recover the full Navier-Stokes equations. In [11], we presented numerical evidence which seems to support that the 3D model develops finite time singularities while the corresponding solution of the 3D Navier-Stokes equations remains smooth. This suggests that the convection term play an essential role in stabilizing the nonlinear vortex stretching term. In this paper, we prove that for any suitable weak solution of the $3 \mathrm{D}$ model in an open set in space-time, the one-dimensional Hausdorff measure of the associated singular set is zero. The partial regularity result of this paper is an analogue of the Caffarelli-Kohn-Nirenberg theory for the 3D Navier-Stokes equations.
\end{abstract}

Keyword: Partial regularity, finite time singularities, 3D Navier-Stokes equations, stabilizing effect of convection.

\section{Introduction}

The question of whether the solution of the 3D Navier-Stokes equations can develop singularities in a finite time from a large smooth initial data with finite energy is one of the most outstanding open mathematical problems [9]. The main difficulty in obtaining the global regularity of the 3D Navier-Stokes equations is due to its super-criticality and the presence of the vortex stretching. Many researchers have contributed to the understanding of the 3D Navier-Stokes equations. The pioneering work was done by Leray in the classical paper [21] in which the author established weak solutions of the 3D Navier-Stokes equations (which is in general called the Leray-Hopf weak solutions due to the important contributions made by Hopf [13] in the case of bounded domains). To understand the regularity properties of the Leray-Hopf weak solutions of the 3D Navier-Stokes equations, Scheffer [27, 28, 29, 30] began

\footnotetext{
*Applied and Comput. Math, Caltech, Pasadena, CA 91125. Email: hou@acm.caltech.edu.

${ }^{\dagger}$ School of Mathematical Sciences, Fudan University, Shanghai 200433, P. R. China; School of Mathematics and Statistics, Northeast Normal University, Changchun 130024, P. R. China; Applied and Comput. Math, Caltech, Pasadena, CA 91125. Email: leizhn@yahoo.com.
} 
the study of localizing Leray's results in space. Scheffer's program was developed further by Caffarelli-Kohn-Nirenberg [2], who proved that the one-dimensional Hausdorff measure of the singularity set is zero. Later, F. Lin [22] gave a simplified proof of the Caffarelli-KohnNirenberg theory (see also [19]). The Caffarelli-Kohn-Nirenberg theory provides an important characterization of the nature of possible singularities of the 3D Navier-Stokes equations. Another important development on the global regularity of the 3D Navier-Stokes equations is to establish some non-blowup criteria for the solution of the 3D Navier-Stokes equations. Many researchers have made important contributions in this area, see, e.g. $[26,32,31,17,1,8]$.

In [11], we studied the stabilizing effect of the convection term for the 3D incompressible Euler and Navier-Stokes equations. We demonstrated the stabilizing effect of convection by constructing a new 3D model for the axisymmetric Navier-Stokes equations with swirl. This model is formulated in terms of a set of new variables related to the angular velocity, the angular vorticity, and the angular stream function. The only difference between our 3D model and the reformulated Navier-Stokes equations in terms of these new variables is that we neglect the convection term in the model. If we add the convection term back to the model, we will recover the full Navier-Stokes equations. This new 3D model preserves almost all the properties of the full 3D Euler or Navier-Stokes equations. In particular, the strong solution of the model satisfies an energy identity similar to that of the full 3D Navier-Stokes equations. We also proved a non-blowup criterion of Beale-Kato-Majda type [1] as well as a non-blowup criterion of Prodi-Serrin type [26, 32] for the model.

Despite the striking similarity at the theoretical level between the $3 \mathrm{D}$ model and the Navier-Stokes equations, our model has a completely different behavior from the full NavierStokes equations. In [11], we provided numerical evidence which seems to support that the model develops finite time singularities from smooth initial data with finite energy. The mechanism for developing these finite time singularities is due to the strong alignment between the variables that contribute to the vortex stretching term. But when we add the convection term back to our model, the mechanism for generating the finite time singularities in the model is destroyed.

In this paper, we study the local behavior of the solutions to the $3 \mathrm{D}$ model equations and establish an analogue of the Caffarelli-Kohn-Nirenberg partial regularity theory for our model. We prove that for any suitable weak solution of the $3 \mathrm{D}$ model in an open set in spacetime, the one-dimensional Hausdorff measure of the associated singular set is zero. The proof of this partial regularity result is similar in spirit to that of Lin in [22], but there are some new technical difficulties associated with our model. One of the difficulties is to handle the singularity induced by the cylindrical coordinates. This makes it difficult to analyze the partial regularity of our model in $\mathbb{R} \times \mathbb{R}^{3}$. To overcome this difficulty, we perform our partial regularity analysis in $\mathbb{R} \times \mathbb{R}^{5}$. By working in $\mathbb{R}^{5}$, we avoid the problem associated with the coordinate singularity.

Another difficulty in obtaining our partial regularity result is that we do not have an evolution equation for the entire velocity field. We need to reformulate our model in terms of a new vector variable. This new variable can be considered as a "generalized velocity field" in $\mathbb{R}^{5}$. We remark that the partial regularity theory for Navier-Stokes equations in $\mathbb{R}^{5}$ is still open due to the lack of certain compactness. For our model reformulated in $\mathbb{R} \times \mathbb{R}^{5}$, we find a 3D structure which has the same scaling as that of the 3D Navier-Stokes equations. This is why the partial regularity analysis can be carried out for the our model in $\mathbb{R} \times \mathbb{R}^{5}$ using a strategy similar to that of Lin in [22]. 
The results presented in this paper and our previous work [11] may have some important implication to the global regularity of the 3D Navier-Stokes equations. We believe that a successful strategy in analyzing the global regularity of the 3D Navier-Stokes equations should take advantage of the stabilizing effect of the convection term in an essential way. So far most of the regularity analyses for the 3D Navier-Stokes equations do not use the stabilizing effect of the convection term. In many cases, the same results can be also obtained for our model. In [11], we have presented numerical evidence which shows that the 3D model is much more singular than the corresponding 3D Navier-Stokes equations. New analytical tools that exploit the local geometric structure of the solution and the stabilizing effect of convection may be needed to prove the global regularity of the 3D Navier-Stokes equations.

We remark that the stabilizing effect of convection has been studied by Hou and Li in a recent paper [10]. They showed that the convection term has a surprising stabilizing effect which cancels the destabilizing term from vortex stretching in a new $1 \mathrm{D}$ model problem. This observation enabled them to obtain a crucial a priori pointwise estimate for a high order norm for the 1D model. Using this a priori estimate, they proved the global regularity of the 3D Navier-Stokes equations for a family of large initial data, whose solutions can lead to large dynamic growth. The stabilizing effect of convection has also been used by Deng-Hou-Yu in $[6,7]$ in deriving localized non-blowup criteria for the 3D Euler equations using a Lagrangian formulation. Recently, Okamoto and Ohkitani [25] investigated the role of the convection term in preventing singularity formation by studying several one-dimensional models and a 2D model derived from the 2D Euler equations.

There has been some interesting development in the study of the $3 \mathrm{D}$ incompressible Navier-Stokes equations and related models. In particular, by exploiting the special structure of the governing equations, Cao and Titi [3] proved the global well-posedness of the 3D viscous primitive equations which model large scale ocean and atmosphere dynamics. For the axisymmetric Navier-Stokes equations, Chen-Strain-Tsai-Yau [4, 5] and Koch-NadirashviliSeregin-Sverak [15] recently proved that if $|u(x, t)| \leq C_{*}|t|^{-1 / 2}$ where $C_{*}$ is allowed to be large, then the velocity field $u$ is regular at time zero.

The rest of this paper is organized as follows. In Section 2 we derive our 3D model and describe some of its important properties. In Section 3, we introduce the notations to be used in this paper. We then give the definition of weak and suitable weak solutions of the 3D model. In Section 4, we establish an important property which asserts that if the generalized velocity field is "sufficiently small" on the unit cylinder $Q_{1}$, then the solution of the 3D model is regular on the smaller cylinder $Q_{\frac{1}{2}}$. In Section 5, we establish a "decay estimate" to study the local behavior of the gradient of the generalized velocity field and prove our partial regularity result.

\section{The derivation of the 3D model and its properties}

In this section, we will give a derivation of the 3D model that we introduced in [11]. Consider the 3D axisymmetric incompressible Navier-Stokes equations with swirl

$$
\left\{\begin{array}{l}
\mathbf{u}_{t}+(\mathbf{u} \cdot \nabla) \mathbf{u}=-\nabla p+\nu \Delta \mathbf{u} \\
\nabla \cdot \mathbf{u}=0, \\
\left.\mathbf{u}\right|_{t=0}=\mathbf{u}_{0}(x), \quad x=\left(x_{1}, x_{2}, z\right)
\end{array}\right.
$$


Let

$$
e_{r}=\left(\frac{x_{1}}{r}, \frac{x_{2}}{r}, 0\right), e_{\theta}=\left(-\frac{x_{2}}{r}, \frac{x_{1}}{r}, 0\right), e_{z}=(0,0,1),
$$

be the three orthogonal unit vectors along the radial, the angular, and the axial directions respectively, $r=\sqrt{x_{1}^{2}+x_{2}^{2}}$. We will decompose the velocity field as follows:

$$
\mathbf{u}=u^{r}(r, z, t) e_{r}+u^{\theta}(r, z, t) e_{\theta}+u^{z}(r, z, t) e_{z},
$$

where $u^{r}, u^{\theta}, u^{z}$ are called the radial, angular and axial velocity respectively. The component $u^{\theta}$ is also referred to as the swirl component of the velocity. One can derive the following axisymmetric form of the Navier-Stokes equations in the cylindrical coordinates [24]:

$$
\left\{\begin{array}{l}
\partial_{t} u^{\theta}+u^{r} \partial_{r} u^{\theta}+u^{z} \partial_{z} u^{\theta}=\nu\left(\Delta_{x}-\frac{1}{r^{2}}\right) u^{\theta}-\frac{u^{r} u^{\theta}}{r} \\
\partial_{t} \omega^{\theta}+u^{r} \partial_{r} \omega^{\theta}+u^{z} \partial_{z} \omega^{\theta}=\nu\left(\Delta_{x}-\frac{1}{r^{2}}\right) \omega^{\theta}+\partial_{z}\left(\frac{\left(u^{\theta}\right)^{2}}{r}\right)+\frac{u^{r} \omega^{\theta}}{r} \\
-\left(\Delta_{x}-\frac{1}{r^{2}}\right) \psi^{\theta}=\omega^{\theta}
\end{array}\right.
$$

where

$$
u^{r}=-\partial_{z} \psi^{\theta}, \quad u^{z}=\frac{1}{r} \partial_{r}\left(r \psi^{\theta}\right) .
$$

The incompressible constraint in cylindrical coordinates is given by

$$
\partial_{r} u^{r}+\partial_{z} u^{z}+\frac{u^{r}}{r}=0 \quad \text { or } \quad \partial_{r}\left(r u^{r}\right)+\partial_{z}\left(r u^{z}\right)=0 .
$$

Introduce the following new variables:

$$
u_{1}=\frac{u^{\theta}}{r}, \quad \omega_{1}=\frac{\omega^{\theta}}{r}, \quad \psi_{1}=\frac{\psi^{\theta}}{r} .
$$

In [10], Hou and Li derived an equivalent reformulation of the Navier-Stokes equations in terms of these new variables as follows:

$$
\left\{\begin{array}{l}
\partial_{t} u_{1}+u^{r} \partial_{r} u_{1}+u^{z} \partial_{z} u_{1}=\nu\left(\partial_{r}^{2}+\frac{3}{r} \partial_{r}+\partial_{z}^{2}\right) u_{1}+2 \partial_{z} \psi_{1} u_{1}, \\
\partial_{t} \omega_{1}+u^{r} \partial_{r} \omega_{1}+u^{z} \partial_{z} \omega_{1}=\nu\left(\partial_{r}^{2}+\frac{3}{r} \partial_{r}+\partial_{z}^{2}\right) \omega_{1}+\partial_{z}\left(\left(u_{1}\right)^{2}\right), \\
-\left(\partial_{r}^{2}+\frac{3}{r} \partial_{r}+\partial_{z}^{2}\right) \psi_{1}=\omega_{1}
\end{array}\right.
$$

where

$$
u^{r}=-\partial_{z}\left(r \psi_{1}\right), \quad u^{z}=\frac{1}{r} \partial_{r}\left(r^{2} \psi_{1}\right)
$$

As observed by Liu and Wang [23], any smooth solution of the Navier-Stokes equations must satisfy the following compatibility condition: $\left.u^{\theta}\right|_{r=0}=\left.\omega^{\theta}\right|_{r=0}=\left.\psi^{\theta}\right|_{r=0}=0$. Thus the variables $u_{1}, \omega_{1}$ and $\psi_{1}$ are well defined.

Our 3D model is derived by simply dropping the convection term from (2.8):

$$
\left\{\begin{array}{l}
\partial_{t} u_{1}=\nu\left(\partial_{r}^{2}+\frac{3}{r} \partial_{r}+\partial_{z}^{2}\right) u_{1}+2 \partial_{z} \psi_{1} u_{1} \\
\partial_{t} \omega_{1}=\nu\left(\partial_{r}^{2}+\frac{3}{r} \partial_{r}+\partial_{z}^{2}\right) \omega_{1}+\partial_{z}\left(u_{1}^{2}\right) \\
-\left(\partial_{r}^{2}+\frac{3}{r} \partial_{r}+\partial_{z}^{2}\right) \psi_{1}=\omega_{1}
\end{array}\right.
$$


Note that (2.10) is already a closed system. The main difference between our 3D model and the original Navier-Stokes equations is that we neglect the convection term in our model. If we add the convection term back to our 3D model, we will recover the Navier-Stokes equations.

This 3D model shares many important properties with the axisymmetric Navier-Stokes equations. First of all, there is an intrinsic incompressible structure in the model equations (2.10). To see this, we define a generalized velocity field as

$$
\begin{aligned}
& \mathbf{u}(t, x)=u^{r}(t, r, z) e_{r}+u^{\theta}(t, r, z) e_{\theta}+u^{z}(t, r, z) e_{z}, \\
& u^{\theta}=r u_{1}, u^{r}=-\left(r \psi_{1}\right)_{z}, u^{z}=\frac{\left(r^{2} \psi_{1}\right)_{r}}{r}
\end{aligned}
$$

where $x=\left(x_{1}, x_{2}, z\right), r=\sqrt{x_{1}^{2}+x_{2}^{2}}, e_{r}, e_{\theta}$ and $e_{z}$ are the three orthogonal unit vectors defined by $(2.2)$. It is easy to check that

$$
\nabla \cdot \mathbf{u}=\partial_{r} u^{r}+\partial_{z} u^{z}+\frac{u^{r}}{r}=0
$$

which is the same incompressibility condition for the original incompressible Euler or NavierStokes equations.

Our model also enjoys the following properties (see [11] for more details and their proofs): i) Energy identity. The strong solution of (2.10) satisfies the following energy identity:

$$
\frac{1}{2} \frac{d}{d t} \int d z \int_{0}^{\infty}\left(\left|u_{1}\right|^{2}+2\left|D \psi_{1}\right|^{2}\right) r^{3} d r+\int d z \int_{0}^{\infty}\left(\left|D u_{1}\right|^{2}+2\left|D^{2} \psi_{1}\right|^{2}\right) r^{3} d r=0,
$$

which has been proved to be equivalent to that of the Navier-Stokes equations. Here $D$ is the first order derivative operator defined in $\mathbb{R}^{5}$, see Section 3 for definition.

ii) A non-blowup criterion of Beale-Kato-Majda type. A smooth solution $\left(u_{1}, \omega_{1}, \psi_{1}\right)$ of the model (2.10) for $0 \leq t<T$ blows up at time $t=T$ if and only if

$$
\int_{0}^{T}\|\nabla \times \mathbf{u}\|_{\mathrm{BMO}\left(\mathbb{R}^{3}\right)} d t=\infty
$$

where $\mathbf{u}$ is defined in (2.11)-(2.12).

iii) A non-blowup criterion of Serrin-Prodi type. A weak solution $\left(u_{1}, \omega_{1}, \psi_{1}\right)$ of the model (2.10) is smooth on $[0, T] \times \mathbb{R}^{3}$ provided that

$$
\left\|u^{\theta}\right\|_{L_{t}^{q} L_{x}^{p}\left([0, T] \times \mathbb{R}^{3}\right)}<\infty
$$

for some $p, q$ satisfying $\frac{3}{p}+\frac{2}{q} \leq 1$ with $3<p \leq \infty$ and $2 \leq q<\infty$.

\section{The main result and suitable weak solutions}

We first state the main result of this paper which is summarized in the following theorem:

Theorem 3.1. For any suitable weak solution of the $3 D$ model equations (2.10) on an open set in space-time, the one-dimensional Hausdorff measure of the associated singular set is zero. 
Before we define the suitable weak solutions of our 3D model, we introduce some notations to be used in this paper. Due to the special nature of the 3D model equations (2.10), we give a slightly different definition of weak and suitable weak solutions from the conventional one. The existence of such weak and suitable weak solutions are sketched at the end of this section.

\subsection{Notations}

Throughout the paper, we often switch our physical space between $\mathbb{R}^{3}$ and $\mathbb{R}^{5}$. We denote by $x=\left(x^{\prime}, z\right)=\left(x_{1}, x_{2}, z\right)$ a point in $\mathbb{R}^{3}$ and $y=\left(y^{\prime}, z\right)=\left(y_{1}, y_{2}, y_{3}, y_{4}, z\right)$ a point in $\mathbb{R}^{5}$. A space-time point in $\mathbb{R} \times \mathbb{R}^{3}$ and $\mathbb{R} \times \mathbb{R}^{5}$ will be denoted by $\xi=(t, x)$ and $\zeta=(t, y)$, respectively. We use $\partial_{t}$ to denote the derivative of a function with respect to time, and $\nabla=\left(\partial_{x^{\prime}}, \partial_{z}\right)^{T}=\left(\partial_{x_{1}}, \partial_{x_{2}}, \partial_{z}\right)^{T}$ and $D=\left(\partial_{y^{\prime}}, \partial_{z}\right)^{T}=\left(\partial_{y_{1}}, \partial_{y_{2}}, \partial_{y_{3}}, \partial_{y_{4}}, \partial_{z}\right)^{T}$ to denote the derivatives of a function with respect to space variables in $\mathbb{R}^{3}$ and $\mathbb{R}^{5}$, respectively. Similarly, we will use Laplacians

$$
\Delta_{x}=\partial_{x_{1}}^{2}+\partial_{x_{2}}^{2}+\partial_{z}^{2}, \quad \Delta_{y}=\partial_{y_{1}}^{2}+\partial_{y_{2}}^{2}+\partial_{y_{3}}^{2}+\partial_{y_{4}}^{2}+\partial_{z}^{2}
$$

Throughout this paper, if the function is axi-symmetric, we will denote its space variable by $(r, z)$, where the $z$-axis is the symmetry axis and $r=\sqrt{x_{1}^{2}+x_{2}^{2}}$ in $\mathbb{R}^{3}$ and $r=$ $\sqrt{y_{1}^{2}+y_{2}^{2}+y_{3}^{2}+y_{4}^{2}}$ in $\mathbb{R}^{5}$, respectively. In particular, for an axi-symmetric function $f=$ $f(r, z)$, we have

$$
\Delta_{y} f=\left(\partial_{r}^{2}+\frac{3}{r} \partial_{r}+\partial_{z}^{2}\right) f
$$

Further, we denote the domain consisting of a ball or a parabolic cylinder as follows:

$$
\left\{\begin{array}{l}
B\left(x_{0}, \theta\right)=\left\{x \in \mathbb{R}^{3}|| x-x_{0} \mid<\theta\right\}, \\
Q\left(\xi_{0}, \theta\right)=\left\{\xi \in \mathbb{R} \times \mathbb{R}^{5}|| x-x_{0} \mid<\theta, t_{0}-\theta^{2}<t<t_{0}\right\}, \\
B\left(y_{0}, \theta\right)=\left\{y \in \mathbb{R}^{5}|| y-y_{0} \mid<\theta\right\}, \\
Q\left(\zeta_{0}, \theta\right)=\left\{\zeta \in \mathbb{R} \times \mathbb{R}^{5}|| y-y_{0} \mid<\theta, t_{0}-\theta^{2}<t<t_{0}\right\} .
\end{array}\right.
$$

In the case when the ball and cylinder are centered at the origin, we will use the following abbreviation: $B_{\theta}=B(0, \theta)$ and $Q_{\theta}=Q(0, \theta)$. Their dimension will be indicated in the context without creating confusion. Further, we will denote the time interval $(0, T)$ by $I$.

We will use $L_{t}^{q} L_{y}^{p}(I \times \Omega)\left(\right.$ resp. $\left.L_{t}^{q} L_{x}^{p}\right)$ to denote the space-time norm

$$
\|f\|_{L_{t}^{q} L_{y}^{p}(I \times \Omega)}=\left[\int_{I}\left(\int_{\Omega}|f|^{p} d y\right)^{q / p} d t\right]^{1 / q}
$$

with the usual modifications when $p$ or $q$ is equal to infinity, or the domain is replaced by $Q\left(\zeta_{0}, \theta\right)$ or $Q\left(\xi_{0}, \theta\right)$. The mean value of a function will be denoted by

$$
\left\{\begin{array}{l}
{[f]_{y_{0}, \theta}=\frac{1}{\left|B\left(y_{0}, \theta\right)\right|} \int_{B\left(y_{0}, \theta\right)} f(t, y) d y} \\
(f)_{\zeta_{0}, \theta}=\frac{1}{\left|Q\left(y_{0}, \theta\right)\right|} \int_{Q\left(y_{0}, \theta\right)} f(t, y) d y
\end{array}\right.
$$

When the domain is $B_{\theta}$ or $Q_{\theta}$, we use the abbreviation $[f]_{\theta}$ or $(f)_{\theta}$, respectively. 


\subsection{Weak and Suitable Weak Solutions}

Let $R>0$ be a constant. We use $\Omega_{x}$ to denote one of the following domains in $\mathbb{R}^{3}$ :

- the whole space $\mathbb{R}^{3}$;

- $\left\{x \in \mathbb{R}^{3} \mid \sqrt{x_{1}^{2}+x_{2}^{2}}<R, z \in \mathbb{R}\right\}$;

- $\left\{x \in \mathbb{R}^{3} \mid \sqrt{x_{1}^{2}+x_{2}^{2}}<R, z \in \mathbb{T}^{1}\right\}, \quad \mathbb{T}^{1}$ is the 1-dimensional torus.

Similarly, we use $\Omega_{y}$ to denote one of the following domains in $\mathbb{R}^{5}$ :

- the whole space $\mathbb{R}^{5}$;

- $\left\{y \in \mathbb{R}^{5} \mid \sqrt{y_{1}^{2}+y_{2}^{2}+y_{3}^{2}+y_{4}^{2}}<R, z \in \mathbb{R}\right\}$;

- $\left\{y \in \mathbb{R}^{5} \mid \sqrt{y_{1}^{2}+y_{2}^{2}+y_{3}^{2}+y_{4}^{2}}<R, z \in \mathbb{T}^{1}\right\}$.

The initial condition for our 3D model is of the form:

$$
u_{1}(0, x)=u_{10}(r, z), \omega_{1}(0, x)=\omega_{10}(r, z), \psi_{1}(0, x)=\psi_{10}(r, z), \quad \text { in } \Omega_{x} .
$$

When we consider the $3 \mathrm{D}$ model in $\mathbb{R}^{5}$, the initial condition becomes

$$
u_{1}(0, y)=u_{10}(r, z), \omega_{1}(0, y)=\omega_{10}(r, z), \psi_{1}(0, y)=\psi_{10}(r, z), \quad \text { in } \Omega_{y} .
$$

In both cases, we require that $\omega_{10}$ and $\psi_{10}$ satisfy the following compatibility condition:

$$
\omega_{10}(r, z)=-\left(\partial_{r}^{2}+\frac{3}{r} \partial_{r}+\partial_{z}^{2}\right) \psi_{10}(r, z) .
$$

Note that for the initial-boundary value problem in a cylindrical domain with $r<R$ and $z \in \mathbb{T}^{1}$, if we let $\widetilde{\psi}_{1}=\psi_{1}-\int_{0}^{1} \psi_{1}(t, R, z) d z$, then $\left(u_{1}, \widetilde{\psi}_{1}, \omega_{1}\right)$ also satisfies $(2.10)$ with $\int_{0}^{1} \widetilde{\psi}_{1}(t, R, z) d z=0$. Thus, without loss of generality, we assume that $\int_{0}^{1} \psi_{1}(t, R, z) d z=0$ in our 3D model equations (2.10) for the initial-boundary value problem in a cylindrical domain with $r<R$ and $z \in \mathbb{T}^{1}$ (if not, we turn to consider the system for $\left(u_{1}, \widetilde{\psi}_{1}, \omega_{1}\right)$ ). We will impose an analogue of the no-slip, no-flow boundary conditions for the generalized velocity field:

$$
\left.\mathbf{u}\right|_{r=R}=0
$$

which is equivalent to

$$
u^{r}=u^{\theta}=u^{z}=0, \quad \text { i.e. } \quad-r \partial_{z} \psi_{1}=r u_{1}=2 \psi_{1}+r \partial_{r} \psi_{1}=0, \quad \text { on } \quad r=R .
$$

Since $\int_{0}^{1} \psi_{1}(t, R, z) d z=0$, it is easy to see that the boundary conditions (3.6) and (3.5) are equivalent to

$$
u_{1}=\psi_{1}=\partial_{r} \psi_{1}=0 \quad \text { on } r=R .
$$

When $\Omega_{x}=\left\{x \in \mathbb{R}^{3} \mid \sqrt{x_{1}^{2}+x_{2}^{2}}<R, z \in \mathbb{R}\right\}$ or $\Omega_{y}=\left\{y \in \mathbb{R}^{5} \mid \sqrt{y_{1}^{2}+y_{2}^{2}+y_{3}^{2}+y_{4}^{2}}\right.$ $<R, z \in \mathbb{R}\}$, we naturally assume that $\psi_{1}$ decays sufficiently fast as $|z| \rightarrow \infty$.

By the choice of the initial and boundary conditions, it is clear that a smooth axisymmetric solution of the $3 \mathrm{D}$ model $(2.10)$ in $\mathbb{R}^{5}$ is the same as the corresponding solution in $\mathbb{R}^{3}$. To avoid dealing with the coordinate singularity, we choose to work in $\mathbb{R}^{5}$ instead of $\mathbb{R}^{3}$. In what follows, we will present our partial regularity analysis in $\mathbb{R}^{5}$.

First, we define the concept of weak solutions of the 3D model equations (2.10). 
Definition 3.2. By a weak solution of the initial-boundary value problem (2.10), (3.2) and (3.7) (or the initial value problem (2.10)-(3.2)) in $[0, T] \times \Omega_{x}$ ), we mean that there exist two axi-symmetric scalar functions $u_{1}, \psi_{1}: \Omega_{y} \rightarrow \mathbb{R}$ such that if we define $\mathbf{v}=\left(u_{1}, D \psi_{1}\right)$, then $u_{1}, \psi_{1}$, and $\mathbf{v}$ satisfy the following conditions:

(a): $\mathbf{v} \in L_{t}^{\infty} L_{y}^{2}\left([0, T] \times \Omega_{y}\right), D \mathbf{v} \in L_{t}^{2} L_{y}^{2}\left([0, T] \times \Omega_{y}\right)$;

(b): $\mathbf{v}$ is weak continuous on $[0, T]$;

(c): the following system holds in the sense of distribution:

$$
\left\{\begin{array}{l}
\partial_{t} u_{1}=2 \partial_{z} \psi_{1} u_{1}+\Delta_{y} u_{1} \\
\partial_{t} D \psi_{1}=D\left(-\Delta_{y}\right)^{-1} \partial_{z}\left(u_{1}^{2}\right)+\Delta_{y} D \psi_{1}
\end{array}\right.
$$

i.e. we have

$$
\left\{\begin{array}{l}
\int_{0}^{T} \int_{\Omega_{y}}\left(-u_{1} \partial_{t} h_{1}-2 \partial_{z} \psi_{1} u_{1} h_{1}+D u_{1} D h_{1}\right) d y d s=0 \\
\int_{0}^{T} \int_{\Omega_{y}}\left(-\partial_{t} h_{2} D \psi_{1}-D\left(-\Delta_{y}\right)^{-1} \partial_{z}\left(u_{1}^{2}\right) h_{2}+\Delta_{y} \psi_{1} D h_{2}\right) d y d s=0
\end{array}\right.
$$

for all axi-symmetric $h_{1}, h_{2} \in C_{0}^{\infty}\left([0, T] \times \Omega_{y}\right)$;

(d): The energy inequality holds for all $t \in[0, T]$ :

$$
\begin{aligned}
& \frac{1}{2} \int_{\Omega_{y}}\left(u_{1}^{2}(t, \cdot)+2\left|D \psi_{1}(t, \cdot)\right|^{2}\right) d y+\int_{0}^{t} \int_{\Omega_{y}}\left(\left|D u_{1}\right|^{2}+2\left|D^{2} \psi_{1}\right|^{2}\right) d y d s \\
& \leq \frac{1}{2} \int_{\Omega_{y}}\left(u_{10}^{2}+2\left|D \psi_{10}\right|^{2}\right) d y .
\end{aligned}
$$

(e): $\lim _{t \rightarrow 0+}\left\|\mathbf{v}(t, \cdot)-\mathbf{v}_{0}\right\|_{L^{2}\left(\mathbb{R}^{5}\right)}=0, \mathbf{v}_{0}=\left(u_{10}, D \psi_{10}\right)$.

The construction of global weak solutions of the 3D model equations (2.10) can be carried out following a standard procedure since we have the energy identity (2.14). We omit the details here and refer the reader to [34]. To study the partial regularity theory, we need to work with a class of weak solutions called suitable weak solutions introduced by Scheffer [27][30]. By a suitable weak solution $\left(u_{1}, \psi_{1}\right)$ to the $3 \mathrm{D}$ model $(2.10)$, we mean a weak solution $\left(u_{1}, \psi_{1}\right)$ of $(2.10)$ which satisfies the following local energy inequality:

$$
\begin{aligned}
& \int_{0}^{T} \int_{\Omega_{y}}\left(\left|D u_{1}\right|^{2}+2\left|D^{2} \psi_{1}\right|^{2}\right) h d y d s \\
& \leq \int_{0}^{T} \int_{\Omega_{y}}\left(\frac{1}{2}\left|u_{1}\right|^{2}+\left|D \psi_{1}\right|^{2}\right)\left(\partial_{t} h+\Delta_{y} h\right) d y d s \\
& \quad-2 \int_{0}^{T} \int_{\Omega_{y}} \psi_{1}\left(u_{1}^{2} \partial_{z} h+D\left(-\Delta_{y}\right)^{-1} \partial_{z}\left(u_{1}^{2}\right) D h\right) d y d s
\end{aligned}
$$

for all $0 \leq h \in C_{0}^{\infty}\left((0, T) \times \Omega_{y}\right)$.

The above definition is motivated by the following consideration. Let $h_{1}, h_{2} \in C_{0}^{\infty}((0, T) \times$ $\left.\Omega_{y}\right)$ and $\left(u_{1}, \omega_{1}, \psi_{1}\right)$ be a smooth solution to our model equations (2.10). Multiplying the first equation of (3.8) by $h_{1} u_{1}$ and then integrating over $I \times \Omega_{y}$, we have

$$
\int_{0}^{T} \int_{\Omega_{y}}\left|D u_{1}\right|^{2} h_{1} d y d s=\int_{0}^{T} \int_{\Omega_{y}}\left[\frac{1}{2}\left|u_{1}\right|^{2}\left(\partial_{t} h_{1}+\Delta_{y} h_{1}\right)+2 \partial_{z} \psi_{1} u_{1}^{2} h_{1}\right] d y d s
$$


for all $0<t \leq T$. Similarly, multiplying the second equation of (3.8) by $h_{2} D \psi_{1}$ and then integrating over $I \times \Omega_{y}$ yields

$$
\begin{gathered}
\int_{0}^{T} \int_{\Omega_{y}}\left|D^{2} \psi_{1}\right|^{2} h_{2} d y d s=\int_{0}^{T} \int_{\Omega_{y}}\left[\frac{1}{2}\left|D \psi_{1}\right|^{2}\left(\partial_{t} h_{2}+\Delta_{y} h_{2}\right)\right. \\
\left.-\partial_{z} \psi_{1} u_{1}^{2} h_{2}-\psi_{1} u_{1}^{2} \partial_{z} h_{2}-\psi_{1} D\left(-\Delta_{y}\right)^{-1} \partial_{z}\left(u_{1}^{2}\right) D h_{2}\right] d y d s
\end{gathered}
$$

for all $0<t \leq T$. Choose $h_{2}=2 h_{1}=2 h$ in (3.12). Adding (3.11) to (3.12) gives the following equality:

$$
\begin{aligned}
\int_{0}^{T} \int_{\Omega_{y}}\left(\left|D u_{1}\right|^{2}+2\left|D^{2} \psi_{1}\right|^{2}\right) h d y d s & =\int_{0}^{T} \int_{\Omega_{y}}\left(\frac{1}{2}\left|u_{1}\right|^{2}+\left|D \psi_{1}\right|^{2}\right)\left(\partial_{t} h+\Delta_{y} h\right) d y d s \\
& -2 \int_{0}^{T} \int_{\Omega_{y}} \psi_{1}\left(u_{1}^{2} \partial_{z} h+D\left(-\Delta_{y}\right)^{-1} \partial_{z}\left(u_{1}^{2}\right) D h\right) d y d s
\end{aligned}
$$

for all axi-symmetric $h \in C_{0}^{\infty}\left((0, T) \times \Omega_{y}\right)$. Inequality (3.10) is a natural generalization of the above equality when we work with weak solutions of our model equations.

As in [2], a consequence of (3.10) and the weak continuity is the following generalized energy inequality

$$
\begin{aligned}
& \frac{1}{2} \int_{\Omega_{y}}\left(u_{1}^{2}+2\left|D \psi_{1}\right|^{2}\right) h(t, \cdot) d y+\int_{0}^{t} \int_{\Omega_{y}}\left(\left|D u_{1}\right|^{2}+2\left|D^{2} \psi_{1}\right|^{2}\right) h d y d s \\
& \leq \int_{0}^{t} \int_{\Omega_{y}}\left[\left(\frac{1}{2}\left|u_{1}\right|^{2}+\left|D \psi_{1}\right|^{2}\right)\left(\partial_{t} h+\Delta_{y} h\right)-2 \psi_{1}\left(u_{1}^{2} \partial_{z} h+D\left(-\Delta_{y}\right)^{-1} \partial_{z}\left(u_{1}^{2}\right) D h\right)\right] d y d s
\end{aligned}
$$

for all axi-symmetric $0 \leq h \in C_{0}^{\infty}\left((0, T) \times \Omega_{y}\right)$.

It is not clear if a weak solution of the Navier-Stokes equations is a suitable weak one. Similarly, we do not know if a weak solution of the 3D model (2.10) is a suitable weak solution either. The existence of suitable weak solutions of the Navier-Stokes equations was studied by Scheffer [28], Caffarelli-Kohn-Nirenberg [2] and F.-H. Lin [22]. Below we give a brief sketch of the construction of suitable weak solutions of the $3 \mathrm{D}$ model equations (2.10). The construction is similar in spirit to that of the Navier-Stokes equations, see [2] and [22].

We will use the following well-known interpolation lemma. A similar interpolation result in $\mathbb{R}^{3}$ has been used extensively in [2], [22] and [19] in proving the partial regularity result of the 3D Navier-Stokes equations.

Lemma 3.3. Let $r>0$ and $B_{r}$ be a ball with radius $r$ in $\mathbb{R}^{5}$. Then there exists a positive constant $C(q)$ independent of $r$ such that for all $f \in H_{0}^{1}\left(B_{r}\right)$ or $f \in H^{1}\left(B_{r}\right)$ with $\int_{B_{r}} f d y=0$, the multiplicative inequality

$$
\|f\|_{L^{q}\left(B_{r}\right)} \leq C(q)\|f\|_{L^{2}\left(B_{r}\right)}^{\frac{5}{q}-\frac{3}{2}}\|D f\|_{L^{2}\left(B_{r}\right)}^{\frac{5}{2}-\frac{5}{q}}
$$

is valid with $q \in\left[2, \frac{10}{3}\right]$. If $f \in H^{1}\left(B_{r}\right)$ with $\int_{B_{r}} f d y \neq 0$, then multiplicative inequality

$$
\|f\|_{L^{q}\left(B_{r}\right)} \leq C(q)\|f\|_{L^{2}\left(B_{r}\right)}^{\frac{5}{q}-\frac{3}{2}}\|D f\|_{L^{2}\left(B_{r}\right)}^{\frac{5}{2}-\frac{5}{2}}+\frac{C(q)}{r^{\frac{5}{2}-\frac{5}{q}}}\|f\|_{L^{2}\left(B_{r}\right)}
$$

is valid with $q \in\left[2, \frac{10}{3}\right]$. 
We will also use the following Sobolev-Poincarè imbedding inequality in our analysis

$$
\|f\|_{L^{5}\left(B_{r}\right)} \leq C\|D f\|_{L^{\frac{5}{2}\left(B_{r}\right)}},
$$

for all $f \in W^{1, \frac{5}{2}}\left(\mathbb{R}^{5}\right)$ satisfying $\int_{B_{r}} f d y=0$.

First of all, we prove the following lemma which is similar in spirit to Theorem 2.2 in [22]:

Lemma 3.4. Let $\left(u_{1}^{(n)}, \psi_{1}^{(n)}\right)$ be a sequence of suitable weak solutions to the 3D model equations (2.10) on $Q_{1}$. Denote $\mathbf{v}^{(n)}=\left(u_{1}^{(n)}, D \psi_{1}^{(n)}\right)$. Assume that $\left(u_{1}^{(n)}, \psi_{1}^{(n)}\right)$ and $\mathbf{v}^{(n)}$ satisfy the following inequality:

$$
\left\|\mathbf{v}^{(n)}\right\|_{L_{t}^{\infty} L_{y}^{2}\left(Q_{1}\right)}+\left\|D \mathbf{v}^{(n)}\right\|_{L_{t}^{2} L_{y}^{2}\left(Q_{1}\right)} \leq C_{0},
$$

for some constant $C_{0}>0$. Further we assume that $\left(u_{1}, D \psi_{1}\right)$ is the weak limit of $\left(u_{1}^{(n)}, D \psi_{1}^{(n)}\right)$ in $L^{2}\left([-1,0], H^{1}\left(B_{1}\right)\right)$. Then $\left(u_{1}, \psi_{1}\right)$ is also a suitable weak solution of the $3 D$ model equations (2.10) on $Q_{1}$.

Proof. For each $n$, since $\left(u_{1}^{(n)}, \psi_{1}^{(n)}\right)$ is a suitable weak solution to the $3 \mathrm{D}$ model equations (2.10) on $Q_{1}$, we have

$$
\begin{aligned}
& \int_{Q_{1}}\left(\left|D u_{1}^{(n)}\right|^{2}+2\left|D^{2} \psi_{1}^{(n)}\right|^{2}\right) h d \zeta \leq \int_{Q_{1}}\left[\left(\frac{1}{2}\left|u_{1}^{(n)}\right|^{2}\right.\right. \\
& \left.\quad+\left|D \psi_{1}^{(n)}\right|^{2}\right)\left(\partial_{t} h+\Delta_{y} h\right)-2 \psi_{1}^{(n)}\left(\left(u_{1}^{(n)}\right)^{2} \partial_{z} h\right. \\
& \left.\left.\quad+D\left(-\Delta_{y}\right)^{-1} \partial_{z}\left[\left(u_{1}^{(n)}\right)^{2}\right] D h\right)\right] d \zeta, \quad \text { for all } 0 \leq h \in C_{0}^{\infty}\left(Q_{1}\right) .
\end{aligned}
$$

Using Fatou's lemma, we can further deduce from the above inequality that

$$
\begin{aligned}
\int_{Q_{1}}\left(\left|D u_{1}\right|^{2}+2\left|D^{2} \psi_{1}\right|^{2}\right) h d \zeta \leq & \liminf _{n \rightarrow \infty} \int_{Q_{1}}\left(\left|D u_{1}^{(n)}\right|^{2}+2\left|D^{2} \psi_{1}^{(n)}\right|^{2}\right) h d \zeta \\
\leq & \liminf _{n \rightarrow \infty} \int_{Q_{1}}\left[\left(\frac{1}{2}\left|u_{1}^{(n)}\right|^{2}+\left|D \psi_{1}^{(n)}\right|^{2}\right)\left(\partial_{t} h+\Delta_{y} h\right)\right. \\
& \left.\quad-2 \psi_{1}^{(n)}\left(\left(u_{1}^{(n)}\right)^{2} \partial_{z} h+D\left(-\Delta_{y}\right)^{-1} \partial_{z}\left[\left(u_{1}^{(n)}\right)^{2}\right] D h\right)\right] d \zeta
\end{aligned}
$$

for all $0 \leq h \in C_{0}^{\infty}\left(Q_{1}\right)$. To estimate the last term on the right hand side of (3.18), we may assume that $\int_{B_{1}} \psi_{1}^{(n)} d y=0$ since

$$
\int_{Q_{1}}\left(\left(u_{1}^{(n)}\right)^{2} \partial_{z} h+D\left(-\Delta_{y}\right)^{-1} \partial_{z}\left[\left(u_{1}^{(n)}\right)^{2}\right] D h\right) d \zeta=0,
$$

which can be proved by performing integration by parts. Using the Hölder inequality, the Calderón-Zygmund theorem and the Sobolev-Poincarè imbedding inequality (3.16), we obtain

$$
\begin{aligned}
& \left|\int_{Q_{1}} \psi_{1}^{(n)}\left(\left(u_{1}^{(n)}\right)^{2} \partial_{z} h+D\left(-\Delta_{y}\right)^{-1} \partial_{z}\left[\left(u_{1}^{(n)}\right)^{2}\right] D h\right) d \zeta\right| \\
& \leq C \int_{-1}^{0}\left\|\psi_{1}^{(n)}\right\|_{L^{5}\left(B_{1}\right)}\left\|u_{1}^{(n)}\right\|_{L^{5 / 2}\left(B_{1}\right)}^{2} d t \\
& \leq C \int_{-1}^{0}\left\|\mathbf{v}^{(n)}\right\|_{L^{5 / 2}\left(B_{1}\right)}^{3} d t=C\left\|\mathbf{v}^{(n)}\right\|_{L_{t}^{3} L_{y}^{5 / 2}\left(Q_{1}\right)}^{3} .
\end{aligned}
$$


If we can prove that $\mathbf{v}^{(n)}$ strongly converges to $\mathbf{v}=\left(u_{1}, D \psi_{1}\right)$ in $L_{t}^{3} L_{y}^{\frac{5}{2}}\left(Q_{1}\right)$, then we can pass the limit inside the integral on the right hand side of (3.18) and the lemma follows.

We now prove the strong convergence of $\mathbf{v}^{(n)}$ in $L_{t}^{3} L_{y}^{\frac{5}{2}}\left(Q_{1}\right)$. Using the assumption $(3.17)$ and the interpolation inequality (3.15) with $q=\frac{5}{2}$, we can show that

$$
\left\|\mathbf{v}^{(n)}\right\|_{L_{t}^{4} L_{y}^{\frac{5}{2}}\left(Q_{1}\right)} \leq C .
$$

Next, we will show that $\partial_{t} \mathbf{v}^{(n)}$ is uniformly bounded in $L_{t}^{2} H_{y}^{-2}\left(Q_{1}\right)$. To see this, let $\phi$ be any smooth, compactly supported test function in $H^{2}\left(B_{1}\right)$. Using (3.8), the Hölder inequality, and performing integration by parts, we obtain

$$
\begin{aligned}
\left|\left(\partial_{t} u_{1}^{(n)}, \phi\right)\right| & =\left|\left(2 u_{1}^{(n)} \partial_{z} \psi_{1}^{(n)}, \phi\right)+\left(\nu u_{1}^{(n)}, \Delta_{y} \phi\right)\right| \\
& \leq 2\left\|u_{1}^{(n)}\right\|_{L_{y}^{5 / 2}\left(B_{1}\right)}\left\|\partial_{z} \psi_{1}^{(n)}\right\|_{L_{y}^{5 / 2}\left(B_{1}\right)}\|\phi\|_{L_{y}^{5}\left(B_{1}\right)}+\nu\left\|u_{1}^{(n)}\right\|_{L_{y}^{2}\left(B_{1}\right)}\|\phi\|_{H^{2}\left(B_{1}\right)} \\
& \leq\left(2\left\|\mathbf{v}^{(n)}\right\|_{L_{y}^{5 / 2}\left(B_{1}\right)}^{2}+\nu\left\|u_{1}^{(n)}\right\|_{L_{y}^{2}\left(B_{1}\right)}\right)\|\phi\|_{H^{2}\left(B_{1}\right)},
\end{aligned}
$$

where we have used the Sobolve imbedding inequality $\|\phi\|_{L^{5}\left(B_{1}\right)} \leq C\|\phi\|_{H^{2}\left(B_{1}\right)}$ in five space dimensions. Similarly, using (3.8) and the Calderón-Zygmund theorem, we can prove that

$$
\left|\left(\partial_{t} D \psi_{1}^{(n)}, \phi\right)\right| \leq C\left(\left\|\mathbf{v}^{(n)}\right\|_{L_{y}^{5 / 2}\left(B_{1}\right)}^{2}+\nu\left\|D \psi_{1}^{(n)}\right\|_{L_{y}^{2}\left(B_{1}\right)}\right)\|\phi\|_{H^{2}\left(B_{1}\right)}
$$

Combining (3.21) with (3.22), we obtain

$$
\left\|\partial_{t} \mathbf{v}^{(n)}\right\|_{H^{-2}\left(B_{1}\right)} \leq C\left(\left\|\mathbf{v}^{(n)}\right\|_{L_{y}^{5 / 2}\left(B_{1}\right)}^{2}+\nu\left\|\mathbf{v}^{(n)}\right\|_{L_{y}^{2}\left(B_{1}\right)}\right)
$$

It follows from (3.20) and (3.17) that $\partial_{t} \mathbf{v}^{(n)}$ is uniformly bounded in $L_{t}^{2} H_{y}^{-2}\left(Q_{1}\right)$. Thus each $\mathbf{v}^{(n)} \in C\left([-1,0], H^{-2}\left(B_{1}\right)\right)$. Now we can apply the well-known compactness theorem (see Theorem 2.1 on pp. 184 in [34] with $X_{1}=H^{-2}\left(B_{1}\right), X_{0}=H^{1}\left(B_{1}\right), X=L^{2}\left(B_{1}\right)$, and $\left.\alpha_{0}=\alpha_{1}=2\right)$ to conclude that $\mathbf{v}^{(n)}$ lies in a compact subset of $L_{t}^{2} L_{y}^{2}\left(Q_{1}\right)$. Using (3.17) and the following Sobolev interpolation inequality

$$
\left\|\mathbf{v}^{(n)}-\mathbf{v}\right\|_{L_{t}^{6} L_{y}^{2}\left(Q_{1}\right)} \leq C\left\|\mathbf{v}^{(n)}-\mathbf{v}\right\|_{L_{t}^{2} L_{y}^{2}\left(Q_{1}\right)}^{\frac{1}{3}}\left\|\mathbf{v}^{(n)}-\mathbf{v}\right\|_{L_{t}^{\infty} L_{y}^{2}\left(Q_{1}\right)}^{\frac{2}{3}},
$$

we conclude that $\mathbf{v}^{(n)}$ (up to a subsequence) converges strongly to $\mathbf{v}$ in $L_{t}^{6} L_{y}^{2}\left(Q_{1}\right)$. On the other hand, the bound (3.17) and the Sobolev interpolation inequality (3.15) with $q=\frac{10}{3}$ give a uniform bound for $\mathbf{v}^{(n)}$ in $L_{t}^{2} L_{y}^{\frac{10}{3}}\left(Q_{1}\right)$. Finally, the following interpolation inequality

$$
\left\|\mathbf{v}^{(n)}-\mathbf{v}\right\|_{L_{t}^{3} L_{y}^{\frac{5}{2}}\left(Q_{1}\right)} \leq C\left\|\mathbf{v}^{(n)}-\mathbf{v}\right\|_{L_{t}^{6} L_{y}^{2}\left(Q_{1}\right)}^{\frac{1}{2}}\left\|\mathbf{v}^{(n)}-\mathbf{v}\right\|_{L_{t}^{2} L_{y}^{\frac{10}{3}}\left(Q_{1}\right)}^{\frac{10}{2}}
$$

implies that $\mathbf{v}^{(n)}$ strongly converges to $\mathbf{v}=\left(u_{1}, D \psi_{1}\right)$ in $L_{t}^{3} L_{y}^{\frac{5}{2}}\left(Q_{1}\right)$. It is easy to show using the strong convergence of $\mathbf{v}^{(n)}$ in $L_{t}^{3} L_{y}^{\frac{5}{2}}\left(Q_{1}\right)$ that $\mathbf{v}$ is a weak solution of the $3 \mathrm{D}$ model equations (2.10). This completes the proof of the lemma. 
Now we consider the following problem which is an approximation to the 3D model. Let $n>0$ be any given integer. We set $\delta=\frac{T}{n}$ and solve the following problem:

$$
\left\{\begin{array}{l}
\partial_{t} u_{1}^{(n)}=2 \partial_{z} \psi_{1}^{(n)} \Phi_{\delta}\left(u_{1}^{(n)}\right)+\Delta_{y} u_{1}^{(n)}, \\
\partial_{t} D \psi_{1}^{(n)}=D\left(-\Delta_{y}\right)^{-1} \partial_{z}\left[u_{1}^{(n)} \Phi_{\delta}\left(u_{1}^{(n)}\right)\right]+\Delta_{y} D \psi_{1}^{(n)},
\end{array}\right.
$$

where $\Phi_{\delta}\left(u_{1}^{(n)}\right)$ is a "retarded mollification" of $u_{1}^{(n)}$, whose value at time $t$ depends only on the values of $u_{1}^{(n)}$ at times prior to $t-\delta$ (see [2]). For fixed $n$, solving (3.24) amounts to solving a linear equation on each strip $m \delta \leq t \leq(m+1) \delta, 0 \leq m \leq n-1$. One can construct suitable weak solutions of (3.24) for each $n$ and prove its weak convergence using a proof similar to that of [2]. By using an argument similar to the proof of Lemma 3.4, we can prove the existence of suitable weak solutions of the $3 \mathrm{D}$ model equations (2.10).

\section{Partial Regularity Theory: Part I}

This and the next section are devoted to proving the partial regularity result of our 3D model. The partial regularity analysis we present here uses a strategy similar to that of [22]. In this section, we prove theorem 4.1 concerning the minimum rate at which a singularity can develop. This is an analogue of Theorem 3.1 in [22]. In Section 5, we will prove theorem 5.1 which is similar to the gradient estimate of Theorem 4.1 in [22]. By using the classical covering lemma and theorem 5.1, we can prove theorem 3.1 in exactly the same as that of the partial regularity theory of the Navier-Stokes equations. We will omit the detail of this part of the proof of theorem 3.1 and refer the reader to [2] for more details. In the remaining part of this paper, we will focus on proving theorem 4.1 and theorem 5.1, which are two key estimates whose analysis is different from that of the Navier-Stokes equations.

Theorem 4.1. There exist two positive constants $\epsilon_{0}$ and $\kappa_{0}$ such that if $\left(u_{1}, \psi_{1}\right)$ is a suitable weak solution of the $3 D$ model equations (2.10) which satisfies

$$
\frac{1}{\theta^{2}} \int_{-\theta^{2}}^{0}\|\mathbf{v}\|_{L^{5 / 2}\left(B_{\theta}\right)}^{3} d t \leq \epsilon_{0}
$$

for some $\theta>0$, then $\mathbf{v}=\left(u_{0}, D \psi_{1}\right)$ is regular at $(0,0)$.

We note that the $3 \mathrm{D}$ model $(2.10)$ has an important scaling property. If $\left(u_{1}, \omega_{1}, \psi_{1}\right)$ is a solution of $(2.10)$, so is

$$
\left(u_{1}^{\lambda}(t, x), \omega_{1}^{\lambda}(t, x), \psi_{1}^{\lambda}(t, x)\right)=\left(\lambda^{2} u_{1}\left(\lambda^{2} t, \lambda x\right), \lambda^{3} \omega_{1}\left(\lambda^{2} t, \lambda x\right), \lambda \psi_{1}\left(\lambda^{2} t, \lambda x\right)\right),
$$

for any $\lambda>0$. Therefore, to prove Theorem 4.1, it suffices to prove

Lemma 4.2. There exist two positive constants $\epsilon_{0}$ and $\kappa_{0}$ such that if $\left(u_{1}, \psi_{1}\right)$ is a suitable weak solution of the $3 D$ model that satisfies

$$
\int_{-1}^{0}\|\mathbf{v}\|_{L^{5 / 2}\left(B_{1}\right)}^{3} d t \leq \epsilon_{0}
$$

then we have

$$
\|\mathbf{v}\|_{C^{\alpha}\left(Q_{\delta}\right)} \leq \kappa_{0}
$$

for some $0<\alpha<1$ and $0<\delta<1$, where $\mathbf{v}=\left(u_{1}, D \psi_{1}\right)$. 
To prove lemma 4.2, we first prove the following lemma:

Lemma 4.3. For given $0<\gamma<\delta \leq \frac{1}{2}$, there exists a positive constant $\epsilon_{0}$ depending only on $\gamma$ such that if $\left(u_{1}, \psi_{1}\right)$ is a suitable weak solution of the $3 D$ model that satisfies

$$
\int_{-1}^{0}\|\mathbf{v}\|_{L^{5 / 2}\left(B_{1}\right)}^{3} d t \leq \epsilon_{0}
$$

then we have

$$
\frac{1}{\gamma^{8}} \int_{-\gamma^{2}}^{0}\left\|\mathbf{v}-(\mathbf{v})_{\gamma}\right\|_{L^{5 / 2}\left(B_{\gamma}\right)}^{3} d t \leq \gamma^{\alpha_{0}} \int_{-1}^{0}\|\mathbf{v}\|_{L^{5 / 2}\left(B_{1}\right)}^{3} d t,
$$

for some positive constant $\alpha_{0} \in\left(0, \frac{1}{5}\right)$, where $\mathbf{v}=\left(u_{1}, D \psi_{1}\right)$ and $(\mathbf{v})_{\gamma}=\frac{1}{\left|Q_{\gamma}\right|} \int_{Q_{\gamma}} \mathbf{v} d \zeta$.

Proof. We will prove the lemma by contradiction. Suppose that the statement of Lemma 4.3 is false. This means that one can find a sequence of suitable weak solutions $\left(u_{1}^{k}, \psi_{1}^{k}\right)$ of the $3 \mathrm{D}$ model equations (2.10) on $Q_{1}$ such that

$$
\int_{-1}^{0}\left\|\mathbf{v}^{k}\right\|_{L^{5 / 2}\left(B_{1}\right)}^{3} d t=\epsilon_{k}^{3} \rightarrow 0 \quad \text { as } k \rightarrow \infty
$$

and

$$
\frac{1}{\gamma^{8}} \int_{-\gamma^{2}}\left\|\mathbf{v}^{k}-\left(\mathbf{v}^{k}\right)_{\gamma}\right\|_{L^{5 / 2}\left(B_{\gamma}\right)}^{3} d t>\gamma^{\alpha_{0}} \int_{-1}^{0}\left\|\mathbf{v}^{k}\right\|_{L^{5 / 2}\left(B_{1}\right)}^{3} d t,
$$

for any $\alpha_{0} \in\left(0, \frac{1}{5}\right)$, where $\mathbf{v}^{k}=\left(u_{1}^{k}, D \psi_{1}^{k}\right)$. Below we will show that (4.5) and (4.6) would lead to a contradiction.

Let

$$
\widetilde{u}_{1}^{k}=\frac{u_{1}^{k}}{\epsilon_{k}}, \quad \widetilde{\omega}_{1}^{k}=\frac{\omega_{1}^{k}}{\epsilon_{k}}, \quad \widetilde{\psi}_{1}^{k}=\frac{\psi_{1}^{k}}{\epsilon_{k}}, \quad \widetilde{\mathbf{v}}^{k}=\frac{\mathbf{v}^{k}}{\epsilon^{k}},
$$

Then $\left(\widetilde{u}_{1}^{k}, \widetilde{\psi}_{1}^{k}\right)$ satisfies

$$
\left\{\begin{array}{l}
\partial_{t} \widetilde{u}_{1}^{k}=\Delta_{y} \widetilde{u}_{1}^{k}+2 \epsilon_{k} \partial_{z} \widetilde{\psi}_{1}^{k} \widetilde{u}_{1}^{k}, \\
\partial_{t} D \widetilde{\psi}_{1}^{k}=D \Delta_{y} \widetilde{\psi}_{1}^{k}+\epsilon_{k} D\left(-\Delta_{y}\right)^{-1} \partial_{z}\left(\left(\widetilde{u}_{1}^{k}\right)^{2}\right), \\
-\Delta_{y} \widetilde{\psi}_{1}^{k}=\widetilde{\omega}_{1}^{k}
\end{array}\right.
$$

in the sense of distribution.

By (4.5), we have

$$
\left\|\widetilde{\mathbf{v}}^{k}\right\|_{L_{t}^{3} L_{y}^{5 / 2}\left(Q_{1}\right)}=1
$$

Consequently, there exists a subsequence of $\left\{\widetilde{\mathbf{v}}^{k}\right\}$ (still denoted by $\left\{\widetilde{\mathbf{v}}^{k}\right\}$ ) such that

$$
\widetilde{\mathbf{v}}^{k}=\left(\widetilde{u}_{1}^{k}, D \widetilde{\psi}_{1}^{k}\right) \rightarrow \widetilde{\mathbf{v}}=\left(\widetilde{u}_{1}, D \widetilde{\psi}_{1}\right) \quad \text { in } \quad L_{t}^{3} L_{y}^{5 / 2}\left(Q_{1}\right)
$$

for some $\widetilde{\mathbf{v}}=\left(\widetilde{u}_{1}, D \widetilde{\psi}_{1}\right) \in L_{t}^{3} L_{y}^{5 / 2}\left(Q_{1}\right)$. Using (4.8)-(4.9) and the Calderón-Zygmund theorem, we can show that the weak limit $\widetilde{\mathbf{v}}$ satisfies the following equations:

$$
\left\{\begin{array}{l}
\partial_{t} \widetilde{u}_{1}=\Delta_{y} \widetilde{u}_{1}, \\
\partial_{t} D \widetilde{\psi}_{1}=D \Delta_{y} \widetilde{\psi}_{1} \\
-\Delta_{y} \widetilde{\psi}_{1}=\widetilde{\omega}_{1}
\end{array}\right.
$$


in the sense of distribution. The classical parabolic estimates imply that $\left(\widetilde{u}_{1}, \widetilde{\psi}_{1}\right)$ is smooth and

$$
\frac{1}{\gamma^{8}} \int_{-\gamma^{2}}^{0}\left\|\widetilde{\mathbf{v}}-(\widetilde{\mathbf{v}})_{\gamma}\right\|_{L^{5 / 2}\left(B_{\gamma}\right)}^{3} d t \leq C \gamma^{2 \alpha_{0}}
$$

for $0<\gamma<\delta \leq \frac{1}{2}$ and some constant $C>0$. By choosing a smaller $\delta$ if necessary, one has

$$
\frac{1}{\gamma^{8}} \int_{-\gamma^{2}}^{0}\left\|\widetilde{\mathbf{v}}-(\widetilde{\mathbf{v}})_{\gamma}\right\|_{L^{5 / 2}\left(B_{\gamma}\right)}^{3} d t \leq \frac{1}{2} \gamma^{\alpha_{0}}
$$

On the other hand, it is easy to see that $\left(\widetilde{u}_{1}^{k}, \widetilde{\psi}_{1}^{k}\right)$ is a suitable weak solution of system (4.8) on $Q_{1}$. In view of (3.19), we may assume that $\int_{B_{1}} \widetilde{\psi}_{1}^{k} d \zeta=0$ without loss of generality. Consequently, by the Sobolev-Poincarè imbedding inequality(3.16) and the Calderón-Zygmund theorem, we obtain

$$
\begin{aligned}
& \left|\int_{-1}^{0} \int_{B_{1}} \widetilde{\psi}_{1}^{(k)}\left(\left(\widetilde{u}_{1}^{(k)}\right)^{2} \partial_{z} h+D\left(-\Delta_{y}\right)^{-1} \partial_{z}\left[\left(\widetilde{u}_{1}^{(k)}\right)^{2}\right] D h\right) d y d s\right| \\
& \leq C \int_{-1}^{0}\left\|\widetilde{\psi}_{1}^{k}\right\|_{L_{y}^{5}\left(B_{1}\right)}\left\|\left|\widetilde{u}_{1}^{k}\right|^{2}\right\|_{L_{y}^{5 / 4}\left(B_{1}\right)} d s \\
& \leq C\left\|\widetilde{\mathbf{v}}^{k}\right\|_{L_{t}^{3} L_{y}^{5 / 2}\left(Q_{1}\right)}^{3} \leq C
\end{aligned}
$$

where we have used (3.16) in the last inequality. Combining (4.13) with the generalized energy inequality (3.13), we get

$$
\left\|\widetilde{\mathbf{v}}^{k}\right\|_{L_{t}^{\infty} L_{y}^{2}\left(Q_{1 / 2}\right)}+\left\|D \widetilde{\mathbf{v}}^{k}\right\|_{L_{t}^{2} L_{y}^{2}\left(Q_{1 / 2}\right)} \leq C,
$$

for some absolute positive constant $C$. Using (4.14) and the dynamic equations (4.8), we can argue exactly as we did in the last part of the proof of Lemma 3.4 to show that $\widetilde{\mathbf{v}}^{k}$ converges strongly to $\widetilde{\mathbf{v}}$ in $L_{t}^{3} L_{y}^{5 / 2}\left(Q_{1 / 2}\right)$, i.e.

$$
\widetilde{\mathbf{v}}^{k} \rightarrow \widetilde{\mathbf{v}} \quad \text { in } \quad L_{t}^{3} L_{y}^{5 / 2}\left(Q_{1 / 2}\right) .
$$

Passing to the limit $k \rightarrow \infty$ in (4.6) gives

$$
\frac{1}{\gamma^{8}} \int_{Q_{\gamma}}\left\|\widetilde{\mathbf{v}}-(\widetilde{\mathbf{v}})_{\gamma}\right\|_{L^{5 / 2}\left(B_{\gamma}\right)}^{3} d t \geq \gamma^{\alpha_{0}}
$$

which contradicts to (4.12). This completes the proof of Lemma 4.3.

Now we are ready to prove Lemma 4.2. Let $\left(u_{1}, \omega_{1}, \psi_{1}\right)$ be a suitable weak solution of the $3 \mathrm{D}$ model equations (2.10). We assume that (4.1) is satisfied. For $0<\gamma<\delta \leq \frac{1}{2}$, we define

$$
\left\{\begin{array}{l}
u_{11}(t, y)=\gamma^{-\alpha_{0} / 3}\left[u_{1}-\left(u_{1}\right)_{\gamma}\right]\left(\gamma^{2} t, \gamma y\right), \\
\omega_{11}(t, y)=\gamma^{1-\alpha_{0} / 3} \omega_{1}\left(\gamma^{2} t, \gamma y\right), \\
\psi_{11}(t, y)=\gamma^{-1-\alpha_{0} / 3}\left(\psi_{1}-\left[\psi_{1}\right]_{\gamma}\right)\left(\gamma^{2} t, \gamma y\right) .
\end{array}\right.
$$


Obviously, $\left(u_{11}, \psi_{11}\right)$ also forms a suitable weak solution of

$$
\left\{\begin{array}{l}
\partial_{t} u_{11}=\left(\partial_{r}^{2}+\frac{3}{r} \partial_{r}+\partial_{z}^{2}\right) u_{11}+2 \gamma^{2} \partial_{z} \psi_{11}\left[\gamma^{\alpha_{0} / 3} u_{11}+\left(u_{1}\right)_{\gamma}\right] \\
\partial_{t} \omega_{11}=\left(\partial_{r}^{2}+\frac{3}{r} \partial_{r}+\partial_{z}^{2}\right) \omega_{11}+\gamma^{2} \partial_{z}\left(\gamma^{\alpha_{0} / 3} u_{11}^{2}+2 u_{11}\left(u_{1}\right)_{\gamma}\right) \\
-\left(\partial_{r}^{2}+\frac{3}{r} \partial_{r}+\partial_{z}^{2}\right) \psi_{11}=\omega_{11}
\end{array}\right.
$$

on $Q_{1}$. Moreover, using Lemma 4.3 and the assumption of Lemma 4.2, we obtain

$$
\begin{aligned}
& \int_{-1}^{0}\left\|\left(u_{11}, D \psi_{11}\right)\right\|_{L^{5 / 2}\left(B_{1}\right)}^{3} d t \\
& =\gamma^{-8-\alpha_{0}} \int_{-\gamma^{2}}^{0}\left\|\left(u_{1}, D \psi_{1}\right)-\left(u_{1}, D \psi_{1}\right)_{\gamma}\right\|_{L^{5 / 2}\left(B_{\gamma}\right)}^{3} d t \\
& \leq \int_{-1}^{0}\|\mathbf{v}\|_{L^{5 / 4}\left(B_{1}\right)}^{3} d t \leq \epsilon_{0} .
\end{aligned}
$$

Applying Lemma 4.3 one more time to $\left(u_{11}, \psi_{11}\right)$, we get

$$
\begin{aligned}
& \frac{1}{\gamma^{8}} \int_{-\gamma^{2}}^{0}\left\|\left(u_{11}, D \psi_{11}\right)-\left(u_{11}, D \psi_{11}\right)_{\gamma}\right\|_{L^{5 / 2}\left(B_{\gamma}\right)}^{3} d t \\
& \leq \gamma^{\alpha_{0}} \int_{-1}^{0}\left\|\left(u_{11}, D \psi_{11}\right)\right\|_{L^{5 / 2}\left(B_{1}\right)}^{3} d t \leq \gamma^{\alpha_{0}} \epsilon_{0},
\end{aligned}
$$

which is equivalent to

$$
\frac{1}{\left(\gamma^{2}\right)^{8}} \int_{-\gamma^{4}}^{0}\left\|\left(u_{1}, D \psi_{1}\right)-\left(u_{1}, D \psi_{1}\right)_{\gamma^{2}}\right\|_{L^{5 / 2}\left(B_{\gamma^{2}}\right)}^{3} d t \leq \epsilon_{0}\left(\gamma^{2}\right)^{\alpha_{0}}
$$

A simple iteration yields that

$$
\frac{1}{\left(\gamma^{k}\right)^{8}} \int_{-\gamma^{2 k}}^{0}\left\|\left(u_{1}, D \psi_{1}\right)-\left(u_{1}, D \psi_{1}\right)_{\gamma^{k}}\right\|_{L^{5 / 2}\left(B_{\gamma^{k}}\right)}^{3} d t \leq \epsilon_{0}\left(\gamma^{k}\right)^{\alpha_{0}},
$$

which gives

$$
\begin{aligned}
& \frac{1}{\left|Q_{\gamma^{k}}\right|} \int_{Q_{\gamma^{k}}}\left|\left(u_{1}, D \psi_{1}\right)-\left(u_{1}, D \psi_{1}\right)_{\gamma^{k}}\right|^{5 / 2} d \zeta \\
& \leq C\left(\frac{1}{\left(\gamma^{k}\right)^{8}} \int_{-\gamma^{2 k}}^{0}\left\|\left(u_{1}, D \psi_{1}\right)-\left(u_{1}, D \psi_{1}\right)_{\gamma^{k}}\right\|_{L^{5 / 2}\left(B_{\gamma^{k}}\right)}^{3} d t\right)^{5 / 6} \\
& \leq C \epsilon_{0}^{5 / 6}\left(\gamma^{k}\right)^{5 \alpha_{0} / 6}
\end{aligned}
$$

for all integer $k \geq 0$. By Campanato's condition, $v=\left(u_{1}, D \psi_{1}\right)$ is Hölder continuous on $Q_{\delta}$. This proves Lemma 4.2. 


\section{Partial Regularity Theory: Part II}

We begin with the following theorem:

Theorem 5.1. Let $\left(u_{1}, \psi_{1}\right)$ be any suitable weak solution of the $3 D$ model equations (2.10) on $Q\left(\zeta_{0}, R_{0}\right)$ and $\mathbf{v}=\left(u_{1}, D \psi_{1}\right)$. There exists a positive constant $\epsilon_{\star}$ such that if

$$
\lim \sup _{\rho \rightarrow 0} \frac{1}{\rho} \int_{Q\left(\zeta_{0}, \rho\right)}|D \mathbf{v}|^{2} d \zeta \leq \epsilon_{\star},
$$

then $\zeta_{0}=\left(t_{0}, y_{0}\right)$ is a regular point of $\left(u_{1}, \psi_{1}\right)$.

As we explained at the beginning of Section 3, the proof of Theorem 3.1 follows by the classical covering lemma and the above Theorem 5.1. We refer the reader to as [2] for details. Below we will present the proof of Theorem 5.1, which is in spirit similar to that of [22].

To prove Theorem 5.1, let us first define several functionals:

$$
\left\{\begin{array}{l}
A_{1}(\theta)=\sup _{t_{0}-\theta^{2} \leq t \leq t_{0}} \frac{1}{\theta} \int_{B\left(y_{0}, \theta\right)}|\mathbf{v}(t, y)|^{2} d y \\
A_{2}(\theta)=\frac{1}{\theta^{2}} \int_{t_{0}-\theta^{2}}^{t_{0}}\|\mathbf{v}(t, \cdot)\|_{L^{5 / 2}\left(B\left(y_{0}, \theta\right)\right)}^{3} d t \\
E(\theta)=\frac{1}{\theta} \int_{Q\left(\zeta_{0}, \theta\right)}|D \mathbf{v}(t, y)|^{2} d \zeta
\end{array}\right.
$$

We need the following lemma:

Lemma 5.2. There holds

$$
A_{2}(\theta) \leq C\left((\theta / \gamma)^{6} A_{1}^{3 / 2}(\gamma)+A_{1}^{3 / 4}(\gamma)(\gamma / \theta)^{3} E^{3 / 4}(\gamma)\right)
$$

for $0<\theta \leq \gamma<R_{0}$, where $C$ is an absolute positive constant.

Proof. First of all, we recall the Poincarè inequality:

$$
\left.\int_{B\left(y_{0}, \gamma\right)}|| \mathbf{v}\right|^{2}-\left[|\mathbf{v}|^{2}\right]_{y_{0}, \gamma}\left|d y \leq C \gamma \int_{B\left(y_{0}, \gamma\right)}\right| \mathbf{v}|| D \mathbf{v} \mid d y .
$$

By using (5.4) and the Hölder inequality, we can show by a straightforward calculation that

$$
\begin{aligned}
\int_{B\left(y_{0}, \theta\right)}|\mathbf{v}|^{2} d y & \leq \int_{B\left(y_{0}, \theta\right)}\left|\left[|\mathbf{v}|^{2}\right]_{y_{0}, \gamma}\right| d y+\left.\int_{B\left(y_{0}, \theta\right)}|| \mathbf{v}\right|^{2}-\left[|\mathbf{v}|^{2}\right]_{y_{0}, \gamma} \mid d y \\
& \leq(\theta / \gamma)^{5} \int_{B\left(y_{0}, \gamma\right)}|\mathbf{v}|^{2} d y+C \gamma \int_{B\left(y_{0}, \gamma\right)}|\mathbf{v}||D \mathbf{v}| d y \\
& \leq(\theta / \gamma)^{5} \gamma A_{1}(\gamma)+C \gamma\left[\gamma A_{1}(\gamma)\right]^{1 / 2}\left(\int_{B\left(y_{0}, \gamma\right)}|D \mathbf{v}|^{2} d y\right)^{1 / 2}
\end{aligned}
$$

Thus, using (5.5) and the standard multiplicative inequality (see Lemma 3.3)

$$
\|\mathbf{v}\|_{L^{\frac{5}{2}\left(B_{\theta}\right)}} \leq C \theta^{-\frac{1}{2}}\|\mathbf{v}\|_{L^{2}\left(B_{\theta}\right)}+C\|\mathbf{v}\|_{L^{2}\left(B_{\theta}\right)}^{\frac{1}{2}}\|D \mathbf{v}\|_{L^{2}\left(B_{\theta}\right)}^{\frac{1}{2}},
$$


we get

$$
\begin{aligned}
& \int_{B\left(y_{0}, \theta\right)}|\mathbf{v}|^{5 / 2} d y \leq \frac{C}{\theta^{5 / 4}}\left(\int_{B\left(y_{0}, \theta\right)}|\mathbf{v}|^{2} d y\right)^{5 / 4} \\
& \quad+C\left(\int_{B\left(y_{0}, \theta\right)}|\mathbf{v}|^{2} d y\right)^{5 / 8}\left(\int_{B\left(y_{0}, \theta\right)}|D \mathbf{v}|^{2} d y\right)^{5 / 8} \\
& \leq \frac{C}{\theta^{5 / 4}}\left[(\theta / \gamma)^{5} \gamma A_{1}(\gamma)+C \gamma\left[\gamma A_{1}(\gamma)\right]^{1 / 2}\left(\int_{B\left(y_{0}, \gamma\right)}|D \mathbf{v}|^{2} d y\right)^{1 / 2}\right]^{5 / 4} \\
& \quad+C \gamma^{5 / 8} A_{1}^{5 / 8}(\gamma)\left(\int_{B\left(y_{0}, \theta\right)}|D \mathbf{v}|^{2} d y\right)^{5 / 8} \\
& \leq C(\theta / \gamma)^{5} A_{1}^{5 / 4}(\gamma)+C A_{1}^{5 / 8}(\gamma)\left(\gamma^{5 / 8}+\gamma^{15 / 8} / \theta^{5 / 4}\right)\left(\int_{B\left(y_{0}, \gamma\right)}|D \mathbf{v}|^{2} d y\right)^{5 / 8} .
\end{aligned}
$$

We finally arrive at

$$
\begin{aligned}
& \int_{t_{0}-\theta^{2}}^{t_{0}}\|\mathbf{v}\|_{L^{5 / 2}\left(B\left(y_{0}, \theta\right)\right)}^{3} d t \leq C \theta^{2}(\theta / \gamma)^{6} A_{1}^{3 / 2}(\gamma) \\
& \quad+C A_{1}^{3 / 4}(\gamma)\left(\gamma^{3 / 4}+\gamma^{9 / 4} / \theta^{3 / 2}\right) \int_{t_{0}-\theta^{2}}^{t_{0}}\left(\int_{B\left(y_{0}, \gamma\right)}|D \mathbf{v}|^{2} d y\right)^{3 / 4} d t \\
& \leq C \theta^{2}(\theta / \gamma)^{6} A_{1}^{3 / 2}(\gamma) \\
& \quad+C A_{1}^{3 / 4}(\gamma)\left(\gamma^{3 / 4}+\gamma^{9 / 4} / \theta^{3 / 2}\right) \theta^{1 / 2}\left(\int_{Q\left(\zeta_{0}, \gamma\right)}|D \mathbf{v}|^{2} d \zeta\right)^{3 / 4} \\
& \leq C \theta^{2}(\theta / \gamma)^{6} A_{1}^{3 / 2}(\gamma)+C A_{1}^{3 / 4}(\gamma)\left(\gamma^{3 / 4}+\gamma^{9 / 4} / \theta^{3 / 2}\right) \theta^{1 / 2} \gamma^{3 / 4} E^{3 / 4}(\gamma) \\
& \leq C \theta^{2}\left[(\theta / \gamma)^{6} A_{1}^{3 / 2}(\gamma)+A_{1}^{3 / 4}(\gamma)(\gamma / \theta)^{3} E^{3 / 4}(\gamma)\right] .
\end{aligned}
$$

This proves the lemma.

We also need the following lemma:

Lemma 5.3. For suitable weak solution $\left(u_{1}, \psi_{1}\right)$ of the $3 D$ model equations $(2.10)$ on $Q_{1}$, we have

$$
A_{1}(\theta / 2)+E(\theta / 2) \leq C\left(A_{2}^{2 / 3}(\theta)+A_{1}(\theta) E^{1 / 2}(\theta)+A_{1}^{1 / 2}(\theta) A_{2}^{1 / 3}(\theta) E^{1 / 2}(\theta)\right),
$$

where $C$ is an absolute positive constant.

Proof. Let $h \in C_{0}^{\infty}\left(Q_{\theta}\right)$ be any smooth cut-off function. Integration by parts gives

$$
\int_{Q_{1}}\left[\psi_{1}\right]_{y_{0}, \theta}\left(u_{1}^{2} \partial_{z} h+D\left(-\Delta_{y}\right)^{-1} \partial_{z}\left(u_{1}^{2}\right) D h\right) d \zeta=0 .
$$

Now we choose a smooth cut-off function $h$ with the following properties:

$$
\left\{\begin{array}{l}
h=0 \text { on } Q\left(\zeta_{0}, \theta\right)^{c}, \quad h=1 \text { in } Q\left(\zeta_{0}, \theta / 2\right) \\
0 \leq h \leq 1, \quad \theta|\nabla h|+\theta^{2}\left(\left|\partial_{t} h\right|+\left|D^{2} h\right|\right) \leq C
\end{array}\right.
$$


Using the generalized energy inequality (3.13) and the Calderón-Zygmund theorem, we get

$$
\begin{aligned}
& A_{1}(\theta / 2)+2 E(\theta / 2) \leq \frac{C}{\theta^{3}} \int_{Q\left(\zeta_{0}, \theta\right)}|\mathbf{v}|^{2} d \zeta \\
& +\frac{C \sup _{t_{0}-\theta^{2}<t<t_{0}}\left|\left[u_{1}^{2}\right]_{y_{0}, \theta}\right|}{\theta^{2}} \int_{t_{0}-\theta^{2}}^{t_{0}} \int_{B\left(y_{0}, \theta\right)}\left|\psi_{1}-\left[\psi_{1}\right]_{y_{0}, \theta}\right| d y d s \\
& +\frac{C}{\theta^{2}} \int_{t_{0}-\theta^{2}}^{t_{0}}\left\|\psi_{1}-\left[\psi_{1}\right]_{y_{0}, \theta}\right\|_{L^{5}\left(B\left(y_{0}, \theta\right)\right)}\left\|\left|u_{1}\right|^{2}-\left[u_{1}^{2}\right]_{y_{0}, \theta}\right\|_{L^{5 / 4}\left(B\left(y_{0}, \theta\right)\right)} d s .
\end{aligned}
$$

We now estimate the three terms on the right hand side of (5.9) one by one. First we use the Hölder inequality to obtain

$$
\frac{1}{\theta^{3}} \int_{Q\left(\zeta_{0}, \theta\right)}|\mathbf{v}|^{2} d \zeta \leq \frac{1}{\theta^{2}} \int_{t_{0}-\theta^{2}}^{t_{0}}\|\mathbf{v}\|_{L^{5 / 2}\left(B\left(y_{0}, \theta\right)\right)}^{2} d t \leq A_{2}^{2 / 3}(\theta) .
$$

Next, we estimate the last term on the right hand side of (5.9). By the Sobolev-Poincaré and the Hölder inequalities, we have

$$
\begin{aligned}
& \frac{1}{\theta^{2}} \int_{t_{0}-\theta^{2}}^{t_{0}}\left\|\psi_{1}-\left[\psi_{1}\right]_{y_{0}, \theta}\right\|_{L^{5}\left(B\left(y_{0}, \theta\right)\right)}\left\|\left|u_{1}\right|^{2}-\left[u_{1}^{2}\right]_{y_{0}, \theta}\right\|_{L^{5 / 4}\left(B\left(y_{0}, \theta\right)\right)} d s \\
& \leq \frac{C}{\theta^{2}} \int_{t_{0}-\theta^{2}}^{t_{0}}\left\{\left\|D \psi_{1}\right\|_{L^{5 / 2}\left(B\left(y_{0}, \theta\right)\right)} \int_{B\left(y_{0}, \theta\right)}\left|u_{1}\right|\left|D u_{1}\right| d y\right\} d s \\
& \leq \frac{C}{\theta^{2}} \int_{t_{0}-\theta^{2}}^{t_{0}}\left\{\theta^{\frac{1}{2}} A_{1}^{1 / 2}(\theta)\|\mathbf{v}\|_{L^{5 / 2}\left(B\left(y_{0}, \theta\right)\right)}\left(\int_{B\left(y_{0}, \theta\right)}|D \mathbf{v}|^{2} d y\right)^{1 / 2}\right\} d t \\
& \leq \frac{C A_{1}^{1 / 2}(\theta)}{\theta^{3 / 2}} \theta^{\frac{1}{3}}\|\mathbf{v}\|_{L_{t}^{3} L_{x}^{5 / 2}\left(Q\left(\zeta_{0}, \theta\right)\right)}\|D \mathbf{v}\|_{L^{2}\left(Q\left(\zeta_{0}, \theta\right)\right)} \leq C A_{1}^{1 / 2}(\theta) A_{2}^{1 / 3}(\theta) E^{1 / 2}(\theta) .
\end{aligned}
$$

Similarly, we can estimate the second term on the right hand side of (5.9) as follows:

$$
\begin{aligned}
& \frac{\sup _{t_{0}-\theta^{2}<t<t_{0}}\left|\left[u_{1}^{2}\right]_{y_{0}, \theta}\right|}{\theta^{2}} \int_{t_{0}-\theta^{2}}^{t_{0}} \int_{B\left(y_{0}, \theta\right)}\left|\psi_{1}-\left[\psi_{1}\right]_{y_{0}, \theta}\right| d y d s \\
& \leq \frac{C A_{1}(\theta)}{\theta^{6}} \int_{t_{0}-\theta^{2}}^{t_{0}} \theta^{5 \times \frac{9}{10}}\left\|\psi_{1}-\left[\psi_{1}\right]_{y_{0}, \theta}\right\|_{L^{10}\left(B\left(y_{0}, \theta\right)\right)} d s \\
& \leq \frac{C A_{1}(\theta)}{\theta^{3 / 2}} \int_{t_{0}-\theta^{2}}^{t_{0}}\left\|D^{2} \psi_{1}\right\|_{L^{2}\left(B\left(y_{0}, \theta\right)\right)} d s \\
& \leq \frac{C A_{1}(\theta)}{\theta^{3 / 2}} \theta\left[\theta^{1 / 2} E^{1 / 2}(\theta)\right] \\
& \leq C A_{1}(\theta) E^{1 / 2}(\theta)
\end{aligned}
$$

The lemma now follows from (5.9)-(5.12).

Proof of Theorem 5.1: Using (5.8) and the inequality $2\left(A_{1}^{1 / 2} E^{1 / 2}\right) A_{2}^{1 / 3} \leq A_{1} E+A_{2}^{2 / 3}$, we get

$$
A_{1}(\gamma \theta / 2) \leq C\left(A_{1}(\gamma \theta) E(\gamma \theta)+A_{2}^{2 / 3}(\gamma \theta)+A_{1}(\gamma \theta) E^{1 / 2}(\gamma \theta)\right) .
$$


For $0<\gamma<1$, it is obvious that

$$
A_{1}(\gamma \theta) \leq \frac{1}{\theta} A_{1}(\gamma), \quad E(\gamma \theta) \leq \frac{1}{\theta} E(\gamma)
$$

Moreover, (5.3) gives

$$
A_{2}(\gamma \theta) \leq C\left(\theta^{6} A_{1}^{3 / 2}(\gamma)+A_{1}^{3 / 4}(\gamma)(1 / \theta)^{3} E^{3 / 4}(\gamma)\right) .
$$

Let $\delta>0$ be sufficiently small such that for all $0<\gamma \leq \delta$ we have $E(\gamma) \leq 2 \epsilon_{\star}$. Using (5.14) and the assumption (5.1) of Theorem 5.1, we obtain for all $0<\gamma \leq \delta$ that

$$
\begin{aligned}
A_{1}(\gamma \theta / 2) & \leq C\left[A_{2}^{2 / 3}(\gamma \theta)+\frac{1}{\theta^{2}} A_{1}(\gamma) E(\gamma)+\frac{1}{\theta^{3 / 2}} A_{1}(\gamma) E^{1 / 2}(\gamma)\right] \\
& \leq C\left[A_{1}(\gamma)\left(\theta^{4}+\frac{1}{\theta^{2}} E(\gamma)+\frac{1}{\theta^{3 / 2}} E^{1 / 2}(\gamma)\right)+\frac{1}{\theta^{8}} E(\gamma)\right] \\
& \leq \frac{1}{2} A_{1}(\gamma)+2 C \epsilon_{\star} / \theta^{8}
\end{aligned}
$$

provided that

$$
\theta \leq \frac{1}{(4 C)^{1 / 4}}, \quad \epsilon_{\star} \leq \min \left\{\frac{\theta^{2}}{16 C}, \frac{\theta^{3}}{128 C^{2}}\right\} .
$$

A simple iterative argument gives

$$
A_{1}\left(\gamma(\theta / 2)^{k+1}\right) \leq \frac{1}{2^{k+1}} A_{1}(\gamma)+4 C \epsilon_{\star} / \theta^{8},
$$

for all integer $k \geq 0$. Now using (5.3) and (5.18), one has

$$
\begin{aligned}
& A_{2}\left(\gamma(\theta / 2)^{k+1}\right) \\
& \leq C\left((\theta / 2)^{6} A_{1}^{3 / 2}\left(\gamma(\theta / 2)^{k}\right)+(2 / \theta)^{3} A_{1}^{3 / 4}\left(\gamma(\theta / 2)^{k}\right) E^{3 / 4}\left(\gamma(\theta / 2)^{k}\right)\right) \\
& \leq C\left(\frac{3}{2}(\theta / 2)^{6} A_{1}^{3 / 2}\left(\gamma(\theta / 2)^{k}\right)+\frac{1}{2}(2 / \theta)^{12} E^{3 / 2}\left(\gamma(\theta / 2)^{k}\right)\right) \\
& \leq \frac{3 C}{2}(\theta / 2)^{6}\left[2^{-3 k / 2} A_{1}^{3 / 2}(\gamma)+\left(4 C \epsilon_{\star}\right)^{3 / 2} / \theta^{12}\right]+\frac{C}{2}(2 / \theta)^{12}\left(2 \epsilon_{\star}\right)^{3 / 2} \\
& \leq \frac{3 C}{2}(\theta / 2)^{6} 2^{-3 k / 2} A_{1}^{3 / 2}(\gamma)+C_{\star} \epsilon_{\star}^{3 / 2},
\end{aligned}
$$

where $C_{\star}=\frac{3 C}{2}(4 C)^{3 / 2} /(2 \theta)^{6}+C \sqrt{2}(2 / \theta)^{12}$.

Now by Theorem $4.1,(0,0)$ is a regular point provided that

$$
\lim \sup _{\rho \rightarrow 0} \frac{1}{\rho^{2}} \int_{-\rho^{2}}^{0}\|\mathbf{v}\|_{L^{5 / 2}\left(B_{\rho}\right)}^{3} d t \leq \epsilon_{0} .
$$

Comparing (5.19) with (5.20), we see that $(0,0)$ is a regular point if we choose $k$ large enough and $\epsilon_{\star}>0$ small enough such that

$$
C_{\star} \epsilon_{\star}^{3 / 2}<\frac{\epsilon_{0}}{2}, \quad \frac{3 C}{2}(\theta / 2)^{6} 2^{-3 k / 2} A_{1}^{3 / 2}(\gamma)<\frac{\epsilon_{0}}{2} .
$$

Choose $\epsilon_{\star}=\min \left\{\left(\frac{\epsilon_{0}}{2 C_{\star}}\right)^{2 / 3}, \frac{\theta^{2}}{16 C}, \frac{\theta^{3}}{128 C^{2}}\right\}$. We see that (5.21) and (5.17) are satisfied. This proves Theorem 5.1. 


\section{Acknowledgement}

The work was in part supported by NSF under the FRG Grant DMS-0353838, ITR Grant ACI-0204932 and DMS-0713670, and China Postdoctoral Science Foundation 20070410160. The authors would like to thank Prof. Congming Li for valuable comments and suggestions.

\section{References}

[1] J. T. Beale, T. Kato and A. Majda, Remarks on the breakdown of smooth solutions for the 3-D Euler equations. Comm. Math. Phys. 94 (1984), no. 1, 61-66.

[2] L. Caffarelli, R. Kohn and L. Nirenberg, Partial regularity of suitable weak solutions of the Navier-Stokes equations, Comm. Pure Appl. Math. 35(1982), 771-831.

[3] C. Cao and E. S. Titi, Global well-posedness of the three-dimensional primitive equations of large scale ocean and atmosphere dynamics, Ann. Math. 166 (2007), no. 1, 245-267.

[4] C. Chen, R. M. Strain, T. Tsai and H. T. Yau Lower bound on the blow-up rate of the axisymmetric Navier-Stokes equations, Int. Math. Res. Not. 2008 (2008), published on March 14, 2008, doi:10.1093/imrn/rnn016.

[5] C. Chen, R. M. Strain, T. Tsai and H. T. Yau Lower bound on the blow-up rate of the axisymmetric Navier-Stokes equations II, arXiv:math.AP/0709.3599.

[6] J. Deng and T. Y. Hou and X. Yu, Geometric properties and non-blowup of 3-D incompressible Euler flow, Commun. PDEs 30 (2005), 225-243.

[7] J. Deng and T. Y. Hou and X. Yu, Improved geometric conditions for non-blowup of 3D incompressible Euler equation, Commun. PDEs 31 (2006), 293-306.

[8] L. Iskauriaza, G. A. Seregin and V. Shverak, $L_{3, \infty}$-solutions of Navier-Stokes equations and backward uniqueness. (Russian) Uspekhi Mat. Nauk 58 (2003), no. 2(350), 3-44; translation in Russian Math. Surveys 58 (2003), no. 2, 211-250

[9] C. Fefferman, http://www.claymath.org/millennium/Navier-Stokes_Equations.

[10] T. Y. Hou and C. Li, Dynamic stability of the 3D axi-symmetric Navier-Stokes equations with swirl, CPAM 61 (2008), no. 5, 661-697.

[11] T. Y. Hou and Z. Lei, On the stabilizing effect of convection in $3 D$ incompressible flows, CPAM, published online on June 10, 2008, DOI: 10.1002/cpa.20254.

[12] T. Y. Hou, Z. Lei and C. Li, Global regularity of the 3D Axi-symmetric Navier-Stokes equations with anisotropic data, accepted by Commun. PDEs, in press.

[13] E. Hopf, Über die Anfangswertaufgabe für die hydrodynamischen Grundgleichungen. Math. Nachr., 4 (1951), 213-231.

[14] K. Kang, Regularity of axially symmetric flows in a half-space in three dimensions. SIAM J. Math. Anal. 35 (2004), no. 6, 1636-1643 
[15] G. Koch, N. Nadirashvili, G. Seregin, V. Sverak, Liouville theorems for the Navier-Stokes equations and applications, arXiv:math.AP/0709.3599.

[16] H. Kozono and Y. Taniuchi, Bilinear estimates in BMO and the Navier-Stokes equations. Math. Z. 235 (2000), no. 1, 173-194.

[17] O. A. Ladyzhenskaya, On uniqueness and smoothness of generalized solutions to the Navier-Stokes equations. Zap. Nauv̆cn. Sem. Leningrad. Otdel. Mat. Inst. Steklov. (LOMI) 5 (1967), 169-185; English transl., Sem. Math. V. A. Steklov Math. Inst. Leningrad 5 (1969), 60-66.

[18] O. A. Ladyzhenskaya, Unique global solvability of the three-dimensional Cauchy problem for the Navier-Stokes equations in the presence of axial symmetry. (Russian) Zap. Nauv̌cn. Sem. Leningrad. Otdel. Mat. Inst. Steklov. (LOMI) 71968 155-177.

[19] O. A. Ladyzhenskaya and G. A. Seregin, On partial regularity of suitable weak solutions to the three-dimensional Navier-Stokes equations. (English summary) J. Math. Fluid Mech. 1 (1999), no. 4, 356-387.

[20] S. Leonardi, J. Málek, J. Nev̌cas and M. Pokorný, On axially symmetric flows in $\mathbf{R}^{3}$. Z. Anal. Anwendungen 18 (1999), no. 3, 639-649.

[21] J. Leray, Sur le mouvement d'un liquide visqueux emplissant l'espace. Acta. Math., 63 (1934), 193-248.

[22] Fang-hua Lin, A new proof of the Caffarelli-Kohn-Nirenberg theorem. Comm. Pure Appl. Math. 51 (1998), no. 3, 241-257.

[23] J. G. Liu and W. C. Wang, Convergence analysis of the energy and helicity preserving scheme for axisymmetric flows, SINUM 44 (2006), no. 6, 2456-2480.

[24] A. J. Majda and A. L. Bertozzi, Vorticity and Incompressible Flow. Cambridge Texts in Applied Mathematics, 27. Cambridge University Press, Cambridge, 2002.

[25] H. Okamoto and K. Ohkitani, On the role of the convection term in the equations of motion of incompressible fluid, J. Phys. Soc. Japan 74 (2005), no. 10, 2737-2742.

[26] G. Prodi, Un teorema di unicità per le equazioni di Navier-Stokes. Ann. Mat. Pura Appl. (4) $481959173-182$.

[27] V. Scheffer, Partial regularity of solutions to the Navier-Stokes equations. Pacific J. Math. 66 (1976), no. 2, 535-552.

[28] V. Scheffer, Hausdorff measure and the Navier-Stokes equations. Comm. Math. Phys. 55 (1977), no. 2, 97-112.

[29] V. Scheffer, The Navier-Stokes equations on a bounded domain. Comm. Math. Phys. 73 (1980), no. 1, 1-42.

[30] V. Scheffer, Boundary regularity for the Navier-Stokes equations. Comm. Math. Phys. 85 (1982), no. 2, 275-299. 
[31] J. Serrin, On the interior regularity of weak solutions of the Navier-Stokes equations. Arch. Rational Mech. Anal. 91962 187-195.

[32] J. Serrin, The initial value problem for the Navier-Stokes equations. Nonlinear Problems, Univ. of Wisconsin Press, Madison, 1963, 69-98.

[33] M. Struwe, On partial regularity results for the Navier-Stokes equations. Comm. Pure Appl. Math. 41 (1988), no. 4, 437-458.

[34] R. Temam, Navier-Stokes Equations, Theory and Numerical Analysis, AMS Chelsea Publishing, 2001.

[35] M. R. Ukhovskii and V. I. Iudovich, Axially symmetric flows of ideal and viscous fluids filling the whole space. Prikl. Mat. Meh. 32 59-69 (Russian); translated as J. Appl. Math. Mech. 321968 52-61.

[36] H. Beirãoda Veiga, A new regularity class for the Navier-Stokes equations in $R^{n}$. (English summary) A Chinese summary appears in Chinese Ann. Math. Ser. A 16 (1995), no. 6, 797. Chinese Ann. Math. Ser. B 16 (1995), no. 4, 407-412. 